\title{
Soft QCD Results from ATLAS and CMS
}

\author{
Claire Gwenlan*, on behalf of the ATLAS and CMS Collaborations \\ University of Oxford \\ E-mail: c.gwenlanlephysics.ox.ac.uk
}

The ATLAS and CMS Collaborations at the LHC have measured a wide range of properties of soft particle production. These include charged particle differential distributions, forward-backward and azimthual correlations, azimuthal ordering of hadrons, event shapes and identified particle production. Many of the measurements can be used to develop and tune Monte Carlo models. The inclusive and diffractive cross sections have been measured using vertex counting, particle tags, and rapidity gaps. In addition, the mean transverse energy flow and its density is measured out to forward rapidities providing a sensitive probe of models of the underlying event and QCD radiation.

The European Physical Society Conference on High Energy Physics -EPS-HEP2013

18-24 July 2013

Stockholm, Sweden

${ }^{*}$ Speaker. 


\section{Introduction}

Soft QCD physics (low momentum transfer, strong force interactions) dominates the total cross section at hadron colliders. Since its description involves non-perturbative QCD it is not well understood, and we must rely on phenomenological models. Improving our understanding and description of such physics is not only of interest in its own right, but is also needed for improved measurements of high-momentum transfer observables, since soft QCD processes contribute to the overall final state, influencing, for example, measurements of inclusive jet cross sections, missing transverse momentum, and isolation cuts. Measurements of total cross sections, particle yields and kinematic distributions are essential for understanding the physics of hadron production, including the relative roles of soft and hard contributions, and for testing, and tuning, our existing Monte Carlo (MC) models, in order to provide the best description of data. These proceedings provide an overview of selected results, from the wealth of soft QCD measurements now available from the ATLAS [1] and CMS [2] Collaborations at the Large Hadron Collider (LHC).

\section{Results}

\subsection{Inclusive Cross Section Measurements}

The total hadronic cross section can be separated into elastic and inelastic components. The LHC experiments are sensitive to a large fraction of the inelastic cross section, where at least one proton loses a fraction of its energy. The inelastic cross section can, itself, be separated into diffractive and non-diffractive processes. Diffractive events are characterised by a large rapidity "gap" (i.e. absence of activity) due to the exchange of a colour singlet object. Diffractive processes have contributions from single diffractive ( $\mathrm{pp} \rightarrow \mathrm{pX})$, double diffractive ( $\mathrm{pp}$ $\rightarrow \mathrm{XY}$ ) and (a smaller proportion of) central diffractive (pp $\rightarrow$ pXp) events.

ATLAS and CMS have both measured the total inelastic cross section $[3,4]$, making minimal experimental requirements to

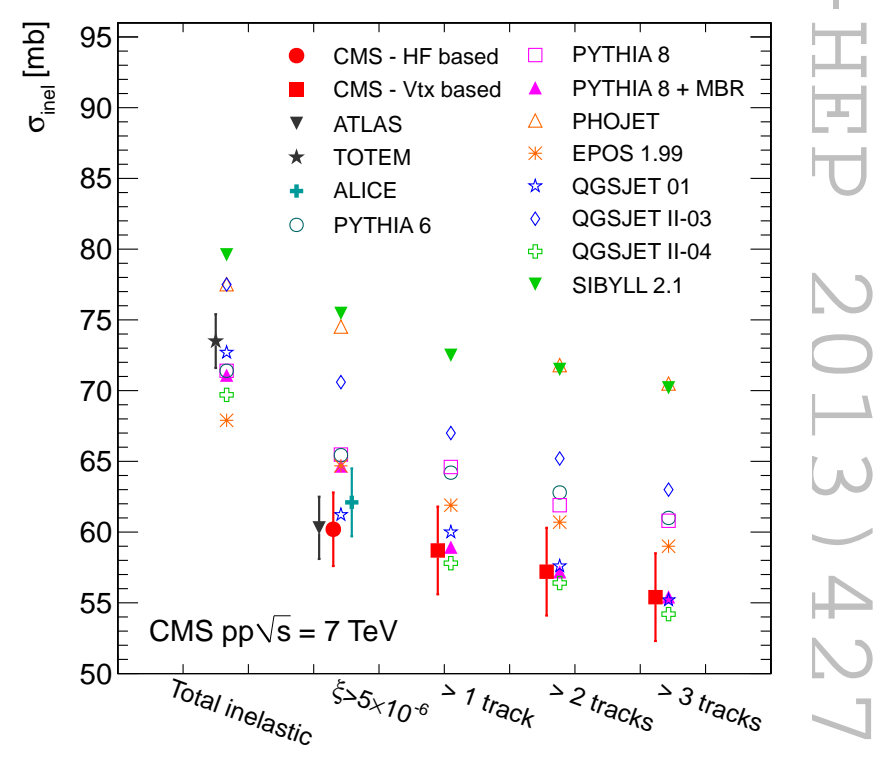

Figure 1: ATLAS and CMS measurements of the inelastic cross section compared to predictions from several MC models, as labelled. Plot from [4]. ensure an inelastic collision has occurred. For ATLAS, two hits in the Minimum Bias Trigger Scintillators (MBTS) are required while, for CMS, events in which $\geq 5 \mathrm{GeV}$ is deposited in the forward hadronic calorimeter, are accepted. The measurements are restricted to the kinematic region $\xi=\left(\mathrm{M}_{\mathrm{X}}^{2}\right) / \mathrm{s}>5 \times 10^{-6}$ (where $\mathrm{M}_{\mathrm{X}}$ is the (highest) invariant mass of particles, selected on one side of the largest rapidity gap in the event), in order to maintain a high efficiency for the minimum bias triggers. The measurements from ATLAS and CMS agree well with each other, as shown in Fig. 1 (black triangle and red circle). 

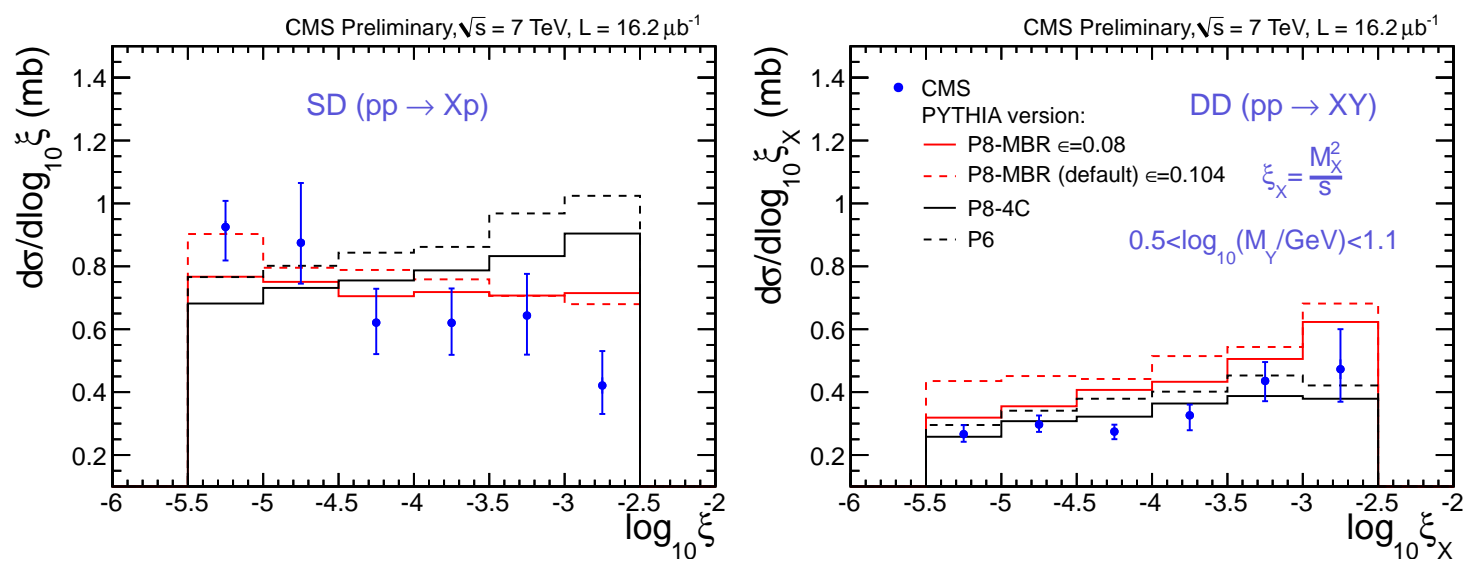

Figure 2: SD (left) and DD (right) cross sections as a function of $\xi=\mathrm{M}_{\mathrm{X}}^{2} / \mathrm{s}$ compared to PYTHIA6, PYTHIA8-4C and PYTHIA8-MBR predictions. Plots from [7].

CMS have also used an alternative technique, counting the number of pileup vertices in an event [4]. This method relies on the assumption that the number of inelastic pp interactions (n), in a given bunch crossing, follows a Poisson distribution, $P(n)=\left(\frac{L \sigma_{\text {inel }}}{n !}\right)^{n} e^{-L \cdot \sigma_{\text {inel }}}$. The data is plotted in bins of instantaneous luminosity, $\mathrm{L}$, for each measured value of $\mathrm{n}$, and fitted with the Poisson distribution to extract values for the total inelastic cross section, $\sigma_{\text {inel }}$. The final results are obtained by averaging the values extracted from each luminosity bin, and are presented as a function of the minimum number of tracks used to build the vertices (1,2 or 3), as shown in Fig. 1 (red squares).

The measurements are compared to a variety of MC models: PYTHIA6 and 8 (which give very similar predictions, independent of tune); PYTHIA8-MBR, with an alternative model of diffraction; and a second class of models based on the same Regge-Gribov phenomenology, but with different implementations of the model ingredients: PHOJET, and three MC models commonly used in cosmic ray physics (EPOS, QGSJET and SIBYLL). The ATLAS and CMS cross section measurements are generally well described by QGSJET01, QGSJET II-04 and PYTHIA8-MBR. Other models tested tend to overestimate the data. Several models, such as PYTHIA6 and 8, are able to reproduce the TOTEM measurement [5] of $\sigma_{\text {inel }}^{\text {total }}$, suggesting these models overestimate the contribution from high mass diffraction and underestimate the low mass component.

ATLAS and CMS have also performed measurements of diffractive cross sections [6, 7]. Both collaborations have measured the total inelastic cross section as a function of $\Delta \eta_{\mathrm{F}}$ (the largest empty pseudorapidity interval, measured from the edge of the detector). The results show the dominance of non-diffractive events at small $\Delta \eta_{\mathrm{F}}$, and the diffractive plateau at large values of $\Delta \eta_{\mathrm{F}}$. Varying the transverse momentum $\left(\mathrm{p}_{\mathrm{T}}\right)$ threshold requirements on the particles entering the measurement, shows that for high $\mathrm{p}_{\mathrm{T}}$, non-diffractive events dominate even out to large values of $\Delta \eta_{\mathrm{F}}$ showing that inclusive events are dominated by low- $\mathrm{p}_{\mathrm{T}}$ production. PYTHIA8 tune $4 \mathrm{C}$ and the PYTHIA8-MBR model give a reasonable description of the data.

CMS have further discriminated between single- (SD) and double-diffractive (DD) events [7] based on topology. Using the CASTOR very forward calorimeter (which covers the pseudorapidity region $\eta=-6.6--5.2)$, SD- and DD-enhanced events have been selected. Activity was required in the central detector and, in addition, activity in CASTOR was either vetoed or required to select SD- and DD-enhanced events, respectively. The results show that PYTHIA8-MBR [8], a Reggebased model with hadronisation tuned to describe diffractive masses at low energies, and including 


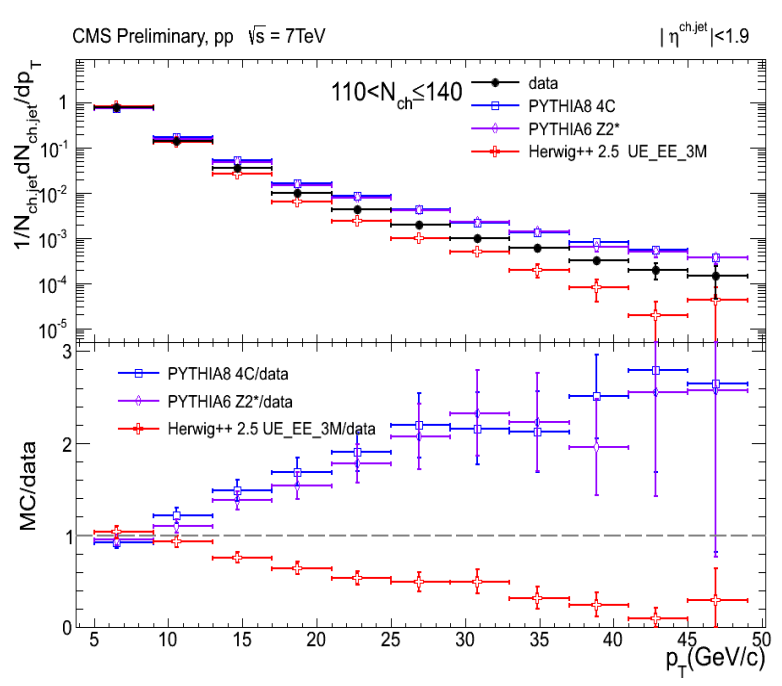

Figure 3: Inclusive charged-particle jet $\mathrm{p}_{\mathrm{T}}$ spectrum at high multiplicity $\left(110<\mathrm{N}_{\mathrm{ch}}<140\right)$, compared to various MC models. Plot from [10].

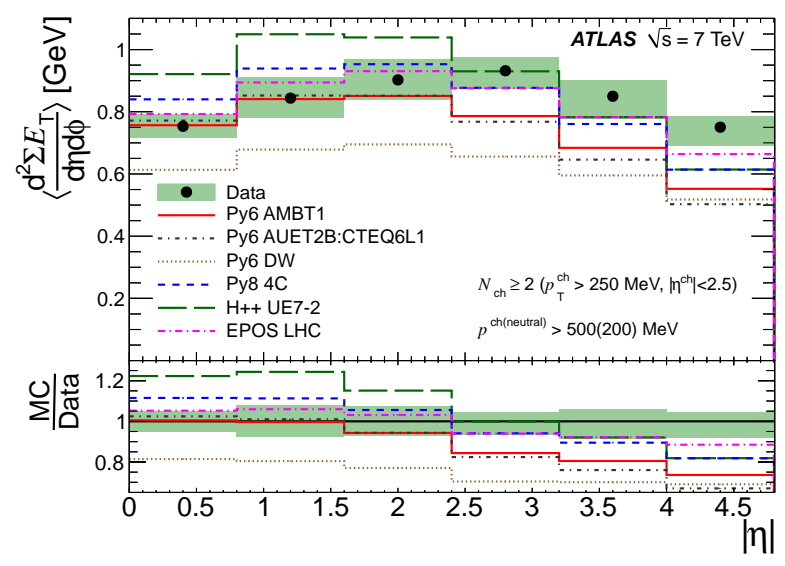

Figure 4: $\mathrm{E}_{\mathrm{T}}$ density compared to various MC models and tunes for the inclusive selection. The filled band represents the total uncertainty on the unfolded data. Plot from [11].

an implementation of the central diffractive process, gives the best description of the data. In particular, the SD-enhanced distribution (Fig 2, left) shows a different trend between the standard PYTHIA (Schuler-Sjöstrand) model of diffraction, and PYTHIA8-MBR. DD cross sections were also measured using only the central detector, by requiring a central rapidity gap, $\Delta \eta>3$.

\subsection{Particle Production}

ATLAS and CMS have performed a wealth of measurements of particle production, including charged particle differential distributions, transverse energy flow, event shapes, particle correlations, and identified particle production. Comparison of such measurements with predictions can highlight areas of discrepancy, and the data can be used to further our understanding and improve the description by the models.

CMS have measured inclusive charged particle production [9] for two different topologies: inclusive and non-single-diffractive (NSD) events. The TOTEM T2 telescopes (triple Gas Electron Multipliers, covering $|\eta|=5.3-6.5, \mathrm{p}_{\mathrm{T}}>40 \mathrm{MeV}$ ), were used to select the events. For the inclusive sample, tracks in $\mathrm{T} 2$ were required in either the forward or backward hemispheres, while for the NSD-enhanced sample, tracks were required in both hemispheres. Measurements were performed in two kinematic regions $\left(\mathrm{p}_{\mathrm{T}}>100 \mathrm{MeV}, \mathrm{p}_{\mathrm{T}}>1 \mathrm{GeV}\right)$ in order to study both soft and hard scattering. The data were compared to a variety of models and tunes: PYTHIA6 Z2*, PYTHIA8 4C, Herwig++ EE3C, EPOS and QGSJET II-04. The level of agreement with the data varies with both $\mathrm{p}_{\mathrm{T}}$ and topology. For the inclusive sample, the PYTHIA6 Z2* tune provides the best description of the data, for both kinematic regions. Most other models overestimate the data for the inclusive topology, though Herwig++ underestimates the measurement for $\mathrm{p}_{\mathrm{T}}>1 \mathrm{GeV}$. For the NSD-enhanced data, no model tested gives a good description of the data. Discrepancies are observed for both topologies, and both kinematic regions, of up to $15 \%$.

CMS have also measured the properties of soft and hard particles as a function of multiplicity [10]. Charged particle tracks were classified as either lying inside (intra-jet) or outside jets, and various properties, such as multiplicity and $\mathrm{p}_{\mathrm{T}}$ distributions of the charged particles, jet rates and 

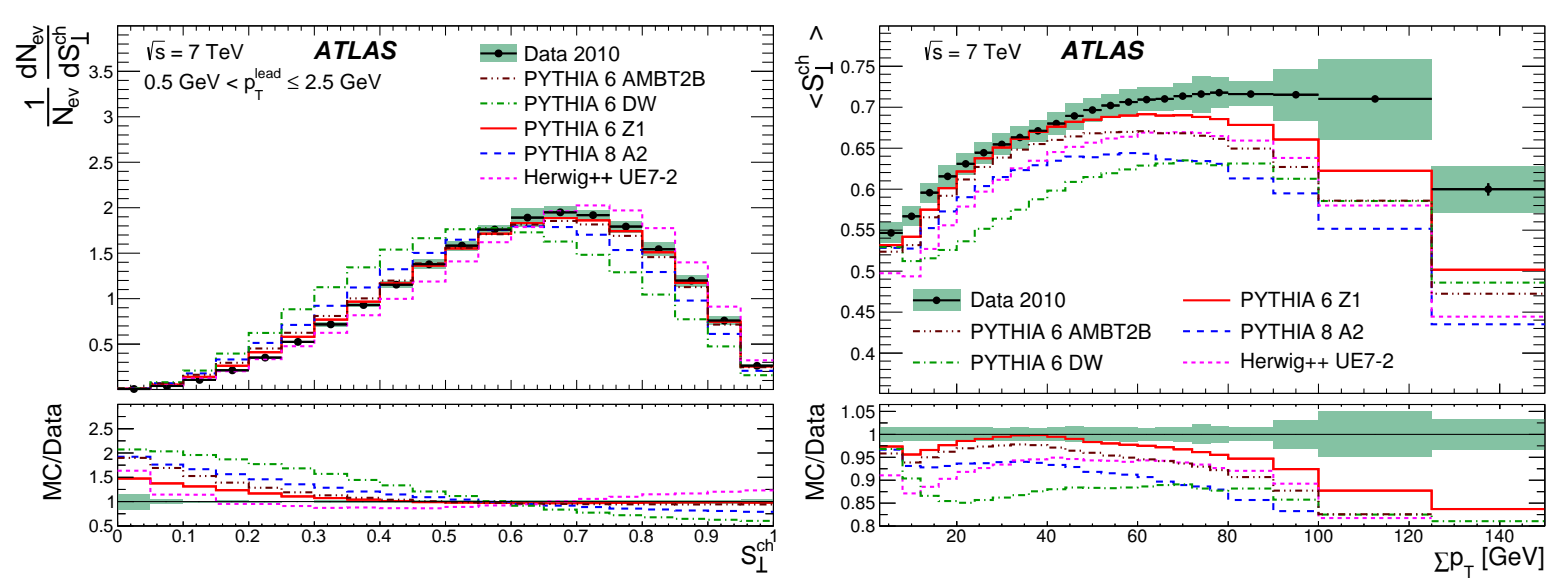

Figure 5: Normalised distribution of the transverse sphericity (left); mean value of the transverse sphericity as a function of $\sum \mathrm{p}_{\mathrm{T}}$ (right). Plots from [12].

jet shapes were measured. Deficiencies in the description of the data were observed, especially at high multiplicities. In particular, the PYTHIA6 Z2* and PYTHIA8 4C models predict too many jets, and the jets are too hard. Herwig++ shows the opposite trend, producing too few jets, that are too soft (see, for example, Fig. 3). The MCs bracket the data, perhaps providing an indication of where the models could be improved.

ATLAS have performed a measurement of the transverse energy flow, and its density, using both charged and neutral particles [11]. The measurement uses the full acceptance of the ATLAS calorimeters, and has been performed for both inclusive (minimum bias) events and in dijet events in the region transverse to the highest- $\mathrm{p}_{\mathrm{T}}$ jet $\left(60^{\circ}<|\phi|<120^{\circ}\right)$, where the distributions are sensitive to the underlying event. The results show that in the central region the data is reasonably well described by recent MC tunes, while in the forward region the models underestimate the data (see, for example, Fig 4, showing the $\mathrm{E}_{\mathrm{T}}$ density for the inclusive selection). Note that the PYTHIA6 DW tune uses only pre-LHC data, and Herwig++ UE7-2 is optimised for the description of the underlying event, so the discrepancies observed with these models also in the central region is not necessarily surprising. Varying the proportion of diffractive events has little effect on the shape of the distributions. Changing the proton PDFs from the default for the analysis, CTEQ6L1, to MSTW08LO (which has a larger gluon density at high- and low-x) provides a better description of the energy flow in the forward region. However, the prediction remains $\sim 15 \%$ below the data at very forward pseudorapidities.

ATLAS have also performed measurements of charged particle event shapes, which were updated for this conference [12]. Event shape variables, such as transverse thrust and transverse sphericity, describe the geometric properties of energy flow in the final state. The measurements show that in inclusive events, which are dominated by low- $\mathrm{p}_{\mathrm{T}}$ production, there is a prevalence of spherical (isotropic) events (see e.g. Fig 5, left). As the $\mathrm{p}_{\mathrm{T}}$ of the leading (highest- $\mathrm{p}_{\mathrm{T}}$ ) particle is increased the events become more jet-like (i.e. the peak of the distribution shifts towards higher values of transverse thrust and lower values of transverse sphericity). The mean value of the event shape variables show an increase as a function of $\sum \mathrm{p}_{\mathrm{T}}$, up to a maximum, and then fall off at very high $\sum \mathrm{p}_{\mathrm{T}}$ (Fig 5, right). The distributions are generally best described by the PYTHIA6 Z2* tune. However, the mean values of the event shape variables, as a function of $\sum \mathrm{p}_{\mathrm{T}}$, show that all models underestimate the data at large $\sum \mathrm{p}_{\mathrm{T}}$. 


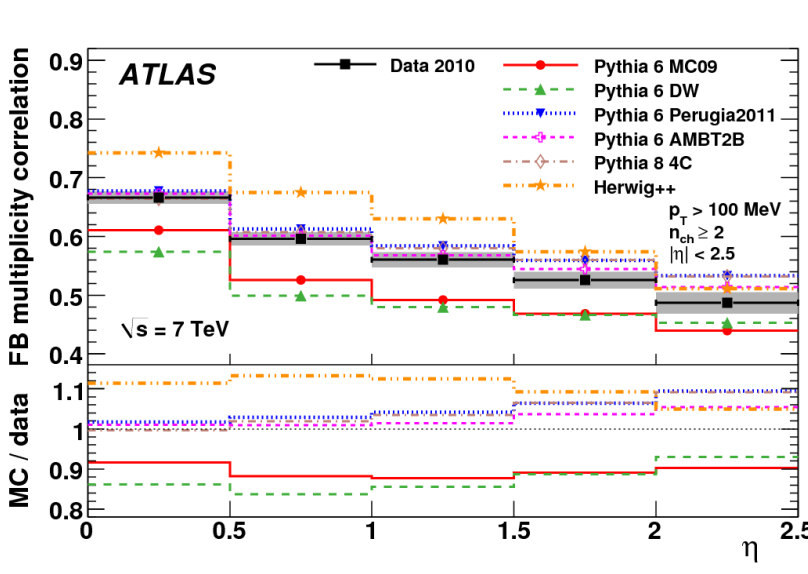

Figure 6: Forward-backward multiplicity correlations in symmetrically opposite $\eta$ intervals, compared to a selection of MC models. Plot from [13].

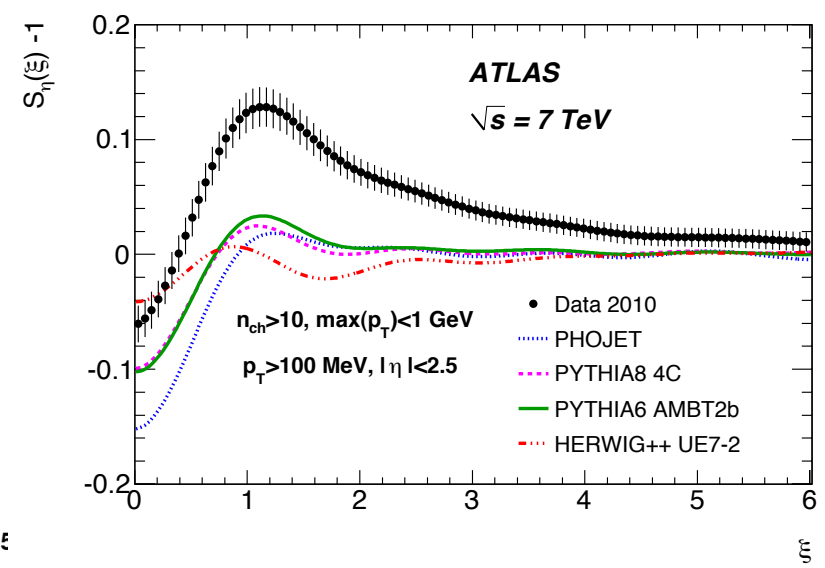

Figure 7: Power spectrum $S_{\eta}(\xi)$, an observable sensitive to the azimuthal ordering of hadrons, compared to various MC models using conventional hadronisation algorithms. Plot from [14].

ATLAS have made a number of recent measurements of charged particle correlations. For example, the so-called forward-backward correlations [13] in which the multiplicity and $\sum \mathrm{p}_{\mathrm{T}}$ correlations are measured in symmetrically opposite $\eta$ regions. The correlation observable is the normalised covariance between the two distributions relative to the mean of each ${ }^{1}$. Measuring such correlations as a function of $\eta$ separation, and particle- $\mathrm{p}_{\mathrm{T}}$, probes both hard and soft contributions, characterised by short-range and long-range correlations, respectively. The results show that the measured correlations can be adequately described by recent MC tunes, which include other LHC data. In particular, the PYTHIA6 AMBT2B tune, which includes this same dataset, though uses different observables, gives a good description of the data. Other recent measurements include charged particle azimuthal distributions [13], azimuthal ordering of hadrons [14] and two-particle correlations [15]. Results show that current MCs have difficulty in describing all features of the available correlation data, tending to over- or underestimate the strength of the correlations. One particular example, is the power spectrum $\mathrm{S}_{\eta}(\xi)$ (an observable sensitive to the azimuthal ordering of hadrons), as shown in Fig. 7, demonstrating the poor description by current models. It has been suggested that alternative hadronisation models might be required in order to describe these data.

Both ATLAS and CMS have produced recent measurements of identified particle production $[16,17]$. ATLAS have measured the spectra of kaons and lambdas, and CMS have studied pion, kaon and proton production. Many features are well described by recent MC tunes. For example, Fig. 8 (left) shows the $\mathrm{p}_{\mathrm{T}}$ spectra of pions, kaons and protons as a function of $\mathrm{p}_{\mathrm{T}}$, demonstrating that the PYTHIA6 Z2* tune describes the data reasonably well except, perhaps, for protons at low- $\mathrm{p}_{\mathrm{T}}$. However, inadequacies in the description of certain features are observed, especially in strange particle production. This is apparent in the kaon-to-pion ratios as a function of $\mathrm{p}_{\mathrm{T}}$ (as shown in Fig 8 (right)), and in lambda distributions at high- $\mathrm{p}_{\mathrm{T}}$. Discrepancies are also observed in the description of proton $\left\langle\mathrm{p}_{\mathrm{T}}\right\rangle$ at high multiplicities, and as a function of $\sqrt{\mathrm{s}}$, where current models tend to underestimate the data.

\footnotetext{
${ }^{1}$ For example, the forward-backward multiplicity correlation is defined as: $\rho_{\mathrm{FB}}^{n}=\frac{\sum x_{\mathrm{F}}^{n} x_{B}^{n}}{N \sigma_{\mathrm{F}}^{n} \sigma_{\mathrm{B}}^{n}}$ where $x_{F(B)}$ indicates the deviation of forward (backward) multiplicities from their mean, $\sigma_{\mathrm{F}(\mathrm{B})}$ is the standard deviation of forward (backward) distributions about their mean, and the sum is over $\mathrm{N}$ events.
} 

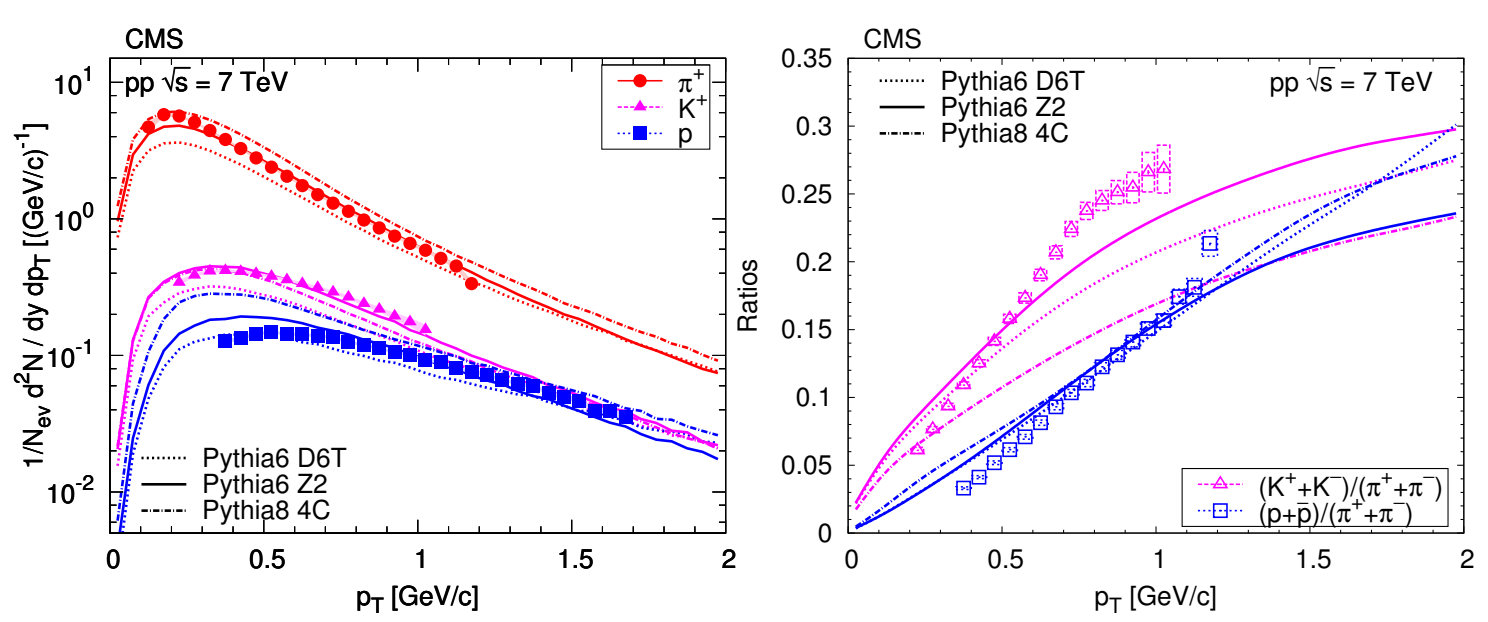

Figure 8: Transverse momentum distributions of identified (positively) charged pions, kaons and protons (left). Ratio of particle yields as a function of transverse momentum (right). Plots from [17]

\section{Summary}

The LHC provides a rich laboratory for studying soft QCD. ATLAS and CMS have provided a wealth of data on event characteristics, particle properties and correlations at the hadron level. Measurements of inelastic cross sections have been made by the experiments using different methods. While many aspects of particle production are adequately described deficiencies are still apparent most especially at high multiplicities, in the forward region, in certain particle correlation measurements, and in strange particle production. The measurements presented here can be used to further our understanding and improve MC models and tuning in the future.

\section{References}

[1] ATLAS Coll., 2008 JINST 3 S08003.

[2] CMS Coll., 2008 JINST 3 S08004.

[3] ATLAS Coll., Nature Commun. 2 (2011) 463.

[4] CMS Coll., Phys. Lett. B 722 (2013) 5.

[5] TOTEM Coll., Eurphys. Lett. 96 (2011) 21002.

[6] ATLAS Coll., Eur. Phys. J. C72 (2012) 1926.

[7] CMS Coll., CMS PAS FSQ-12-005, https://cds.cern.ch/record/1547898.

[8] R. Ciesielski and K. Goulianos, arXiv:1205.1446.

[9] CMS Coll., CMS PAS FSQ-12-026, https://cds.cern.ch/record/1546365.

[10] CMS Coll., CMS PAS FSQ-12-022, https://cds.cern.ch/record/1543202.

[11] ATLAS Coll., JHEP 11 (2012) 033.

[12] ATLAS Coll., Phys. Rev. D88 (2013) 032004.

[13] ATLAS Coll., JHEP 1207 (2012) 019.

[14] ATLAS Coll., Phys. Rev. D86 (2012) 052005.

[15] ATLAS Coll., JHEP 1205 (1212) 157.

[16] ATLAS Coll., Phys. Rev. D85 (2012) 012001.

[17] CMS Coll., Eur. Phys. J. C72 (2012) 2164. 